\title{
RESIDENCY PROGRAM \\ A NEW ERA IN POSTGRADUATE MEDICAL EDUCATION IN BANGLADESH
}

Medical education is the science behind teaching and learning in medicine has been firmly established as a separate discipline. It has gained tremendous progress as a discipline parallel to medical science. It achieved a phase where it is not limited to the understanding of fault but know how to correct these fault. So medical education has converted itself from the role of problem identifier to that of solution provider.

Today the world is progressing not only in arts and culture but also astonishing improvement has been achieved in global health education through the medical education establishment. There is two contradictory view of medical schools. One group thinks that they are producing best physician by successfully cultivating best mind. Other group differ's that medical schools are remarkably far behind to adopting the science of medical education advancement. Some says that both views are true, medical schools are creating the best physicians but at the same time they are remarkably resistant to adopting new changes in learning and teaching paradigms.

Why are the medical faculty and medical schools so reluctant to change? One of the most important factors is the fear of unknown - a substantial lack of knowledge about the science and art of teaching and learning in medicine. Ignorance breeds fear and fear perpetuates the collective inertia. Medical education in Bangladesh was running with the mixture of education in Europe and America. We were following two system one is undergraduate and Postgraduate education system. After MBBS the student must enrolled in post graduate by appearing in a admission test. The postgraduate was run by the then IPGM\&R and BCPS. Most medical teacher were taught in an era of non structured medical education. Teaching was mostly teacher dominated. There was little or no chance of interaction between the teacher and student.

In 2010, the government decided to introduce an unique system in postgraduate education in Bangabandhu Sheikh Mujib Medical University and named as Residency Program. The overall concept of this new way of medical education to meet the three criteria of a. Understandability b. Portability and $c$. Affordability. This training and teaching programme will lead a medical student to become not only a good physician but a good human who care and share the health care system of the nation, by becoming a self directed and lifelong learner.

The objectives: after completion of the course, the student will be able to be a

1. Medical expert

2. Health advocator

3. Communicator

4. Collaborator

5. Manager

6. Scholar

7. Health professional

The student after the enrolment in Residency after a competitive entrance examination will spend five years in respective institute in different phase and blocks. The phase composed of two years in Phase- $A$ and three years in Phase -B. In phase- $A$ they will do on duty learner in different blocks, consist of 1-3 months accordingly to the demand of parent subject in rotatory basis. After each three months they will appear block ending examination to go to the next block. If they pass all the blocks in Phase-A will be eligible for appearing Phase A final examination to go to Phase- 
B. In Phase- B they will stay in their parents department and appear six block ending examination and prepare a thesis sitting for final exam. They must appear thesis before appearing clinical final examination. After passing thesis and clinical examination they will be awarded the degree name MD for medicine faculty and MS for surgical faculty.

This new system has been introduced first in the era of Late Professor M.A. Quadery, while he was the first Vice chancellor of this university. The dream was not fulfilled and somehow interrupted in next period but it is implemented in the time of Professor Pran Gopal Dutta while he was the Vice chancellor of BSMMU. I can't conclude this writing without remember the name of following personalities who were the lead group for Residency in BSMMU like Professor Mohammad Saiful Islam, Professor Syed Atiqul Haque, Professor Nazrul Islam, Professor Manzare Shamim, Professor Chowdhury Ali Kawser, Professor Abdul Jalil and others.

The initial stage is over, the programme is running smoothly with some pitfalls in this university as well as in different medical colleges in Bangladesh. BSMMU is conducting the role as a co-ordinating body. The Entrance examination, phase-A exit examination all are being conducted by university now. It is a difficult task, but we are hopeful.

Now time has not yet come to evaluate the whole programme in 15 years time, but we must prepare our self to coup with this new concept of teaching and learning. So that we can make a new generation physician who will not only fulfilled the demand of the nation but also will match with the whole world. We are waiting for those days.

Prof. Md. Tosaddeque Hossain Siddiqui

Department of Paediatric Surgery

Bangabandhu Sheikh Mujib Medical University

Dhaka, Bangladesh

E-mail: t_siddiqui_2000@yahoo.com

\section{References}

1. Basics in medical education, Zubair Amin, Khoo Hoon Eng National University Singapore.

2. Residency program (curriculum Phase-A), Faculty of Surgery, BSMMU. 